\title{
Localization of Executive Functions in Dual-Task Performance with fMRI
}

\author{
André J. Szameitat ${ }^{1}$, Torsten Schubert ${ }^{2}$, Karsten Müller ${ }^{1}$, and \\ D. Yves von Cramon ${ }^{1}$
}

\begin{abstract}
We report a study that investigated the neuroanatomical correlates of executive functions in dual-task performance with functional magnetic resonance imaging. Participants performed an auditory and a visual three-choice reaction task either separately as single tasks or concurrently as dual tasks. In the dual-task condition, two stimuli were presented in rapid succession to ensure interference between the component tasks (psychological refractory period). The behavioral data showed considerable performance decrements in the dual-task compared to the single-task condition. Dual-task-related activation was detected with two different neuroimaging methods. First, we determined dual-task-related activation according to the method of cognitive subtraction. For that purpose, activation in the dual-task was compared directly with activation in the single-task conditions. This analysis revealed
\end{abstract}

that cortical areas along the inferior frontal sulcus (IFS), the middle frontal gyrus (MFG), and the intraparietal sulcus (IPS) are involved in dual-task performance. The results of the subtraction method were validated with the method of parametric manipulation. For this purpose, a second dual-task condition was introduced, where the difficulty of the dual-task coordination was increased compared with the first dual-task condition. As expected, behavioral dual-task performance decreased with increased dual-task difficulty. Furthermore, the increased dual-task difficulty led to an increase of activation in those cortical regions that proved to be dual-task related with the subtraction method, that is, the IFS, the MFG, and the IPS. These results support the conclusion that dorsolateral prefrontal and superior parietal cortices are involved in the coordination of concurrent and interfering task processing.

\section{INTRODUCTION}

Executive functions are believed to be a prerequisite for complex human behavior, especially in situations where contradictory and interfering information has to be processed in order to execute goal-oriented behavior (Badgaiyan, 2000; Miller, 2000; Knight, Grabowecky, \& Scabini, 1995). One classical example of such situations is dual-task performance, in which two tasks have to be carried out concurrently. Here, executive functions are needed in order to coordinate the concurrent processing of the different streams of information. Accordingly, the investigation of dual-task performance gives insights into how the brain realizes complex human behavior.

However, the precise functioning and neural implementation of these executive functions is still unresolved. Previous neuroimaging studies investigating dual-task performance yielded rather contradictory results with respect to the functional neuroanatomy of dual-task processing. While some studies identified the lateral prefrontal cortex as related to dual-task perfor-

\footnotetext{
${ }^{1}$ Max-Planck-Institute of Cognitive Neuroscience, Leipzig,

${ }^{2}$ Humboldt University, Berlin
}

mance (Herath, Klingberg, Young, Amunts, \& Roland, 2001; Koechlin, Basso, Pietrini, Panzer, \& Grafman, 1999; Goldberg et al., 1998; D'Esposito et al., 1995), other studies failed to show such results (Adcock, Constable, Gore, \& Goldman-Rakic, 2000; Bunge, Klingberg, Jacobsen, \& Gabrieli, 2000; Klingberg, 1998). One reason for these contradictory findings might be the paradigms employed. Previous studies often used rather complex paradigms, which do not allow for control of the processing strategies applied by the participants. Therefore, it is possible that in some of these studies, the participants processed the tasks without interference. Crucially, interference between the processing of the tasks is thought to be the main cause for the need of executive functions in dual-task performance (Meyer \& Kieras, 1997; DeJong, 1995; Umiltà, Nicoletti, Simion, Tagliabue, \& Bagnara, 1992; Baddeley, 1990). Accordingly, if previously used paradigms enabled task processing without interference, executive functions would have been absent (Pashler, 1994; Broadbent, 1982; Craik, 1947). This assumption is supported by the finding that some of the abovementioned studies, which yielded no dualtask-related activation, showed no (Bunge et al., 2000) or rather small (Adcock et al., 2000) performance decrements in the dual-task compared to the single-task 
conditions (see also Smith et al., 2001). Thus, the first aim of the present study was to test for dual-task-related activation by using a behavioral dual-task paradigm, which ensures the presence of interference and, therefore, of executive functions.

A second issue in this study concerns the methodology used to obtain dual-task-related activation. Most previous studies used the method of cognitive subtraction to obtain dual-task-related activation, where dual-task-related activation is detected by subtracting activation in two single tasks from activation in a dual task. However, this approach has been criticized because of some problematic methodological presumptions (Sartori \& Umiltà, 2000; Sidtis, Strother, Anderson, \& Rottenberg, 1999; Braver et al., 1997; Friston, Price, et al., 1996; Sternberg, 1969). As will be shown later, the use of the cognitive subtraction method might have obscured dual-task-related activation so far. Therefore, in the present study, we used the method of parametric manipulation (Braver et al., 1997; Cohen et al., 1997) in addition to the method of cognitive subtraction to assess dual-task-related activation.

\section{Interference in Dual-Task Situations}

The typical indicators of interference in dual-task performance are performance decrements reflected by prolonged reaction times (RTs) or increased error rates, as compared to single-task performance. Theories of dual-task performance have suggested that the interference between two tasks results from a processing mechanism that is limited to processing only one task at a time. According to many authors, such a processing mechanism constitutes a bottleneck during the concurrent processing of two tasks (see Figure 1; Schubert, 1999; Meyer \& Kieras, 1997; Pashler, 1994; DeJong 1993). If the component tasks are processed closely in time, so that they compete for the processing by the bottleneck mechanism, interference arises, which has to be resolved by additional executive functions. In more detail, empirical findings from experimental psychology suggest that in such situations, the processing of one task is interrupted as long as the bottleneck mechanism is processing the other task (Pashler, 1994). ${ }^{1}$ In this context, executive function is conceptualized as coordination of interfering

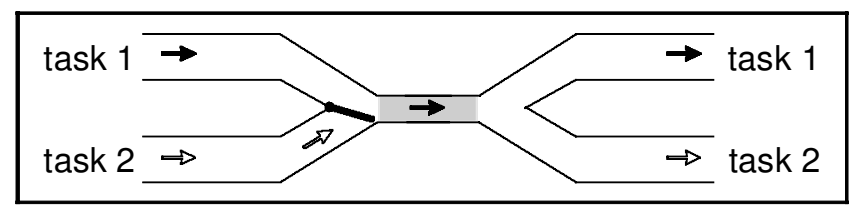

Figure 1. Illustration of the dual-task situation according to the PRP paradigm. Executive functions are involved, because order of information processing in both tasks has to be coordinated at the bottleneck (shaded gray). processing at the stage of the bottleneck by scheduling the order in which the tasks are processed, by interrupting one of the two tasks, switching to the interrupted task, and reinstating the interrupted task when bottleneck processing in the other task has finished (Meyer \& Kieras, 1997; DeJong, 1995). Because the executive functions are required solely in the dualtask but not in the single-task situation, they should lead to increased neural activity, which should be measurable by means of functional magnetic resonance imaging (fMRI).

In the present study, we used the dual-task paradigm of the psychological refractory period (PRP), because it permits assessing the occurrence of a bottleneck mechanism and, accordingly, of executive functions. In this paradigm, two stimuli are presented in rapid succession, separated by a variable stimulus onset asynchrony (SOA), and the participants have to respond to each stimulus in the order of their presentation. The presence of a bottleneck is indicated by the so-called PRP effect, which is reflected by an increase in RT on the second task with decreasing SOA, and by a constant RT on the first task (e.g., Pashler, 1994). When the SOA is short, both tasks temporally overlap considerably and bottleneck processing of the first task leads to an interruption of second task processing, which results in prolonged RTs for the second task. In contrast, when the SOA is long, the temporal overlap of the component tasks is small and, accordingly, the second task is interrupted for a shorter period, which results in shorter RTs for the second task. In brief, this pattern of PRP RTs indicates the presence of concurrent and interfering processes in both tasks.

In the present experiment, participants had to perform a three-choice reaction task on the pitch of an auditory stimulus with their left hand and a three-choice reaction task on the position of a visual stimulus with their right hand, respectively (Figure 6). The order of the component tasks in one of two dual-task conditions was fixed throughout a block. We call this the DT-fixed condition. (In addition to the DT-fixed condition, a second dual-task condition was also administered to the participants, which will be explained below.) Besides the dual-task condition blocks, participants performed the component tasks separately (single tasks; auditory (AUD) and visual (VIS)) and a resting baseline condition (BASE). All conditions were presented blockwise. Based on the cognitive subtraction method (Friston et al., 1995), dual-task-related activation was assessed by testing whether both tasks interact with each other when performed concurrently. ${ }^{2}$ In other words, we tested for overadditive, that is, surplus, activation in the dual-task condition, as compared to the summed activation evoked by the single-task conditions (Koechlin et al., 1999; Friston et al., 1995; Friston, Price, et al., 1996). This comparison takes into account that areas identified as dual-task-related may already be involved in single-task 
processing but are more strongly involved in dual-task processing.

\section{Parametric Manipulation of Dual-Task-Related Executive Functions}

However, the cognitive subtraction method is based on a problematic assumption, that is, the assumption of pure insertion (Donders, 1868/1969). This assumption holds that the insertion of an additional process into a task does not change the remaining processes, an issue that has been questioned by a number of researchers (Sartori \& Umiltà, 2000; Sidtis et al., 1999; Braver et al., 1997; Friston, Price, et al., 1996; Sternberg, 1969). For example, the insertion of an additional task in order to create a dual-task situation may not only insert additional executive functions, but may also change the general perceptual, memory, and motor processes of the first task as well. Consequently, dual-task-related activation revealed by DT-fixed cannot unequivocally be interpreted because it might be caused either by altered component-task processing or by the involvement of additional executive functions in dual-task performance. One way to circumvent such interpretational problems is to use additional information yielded by an alternative methodological approach, the parametric manipulation method (Braver et al., 1997; Cohen et al., 1997). According to this method, one experimental factor that affects the operation of a single process is varied, and it is determined whether this manipulation results in systematic activation changes of certain cortical areas. If this is the case, then these cortical areas can be attributed to the manipulated process.

To implement the parametric manipulation method in the present experiment, a second dual-task condition was introduced, which incorporated generally higher demands on the dual-task-related executive functions than DT-fixed. In this dual-task condition, the presentation order of the component tasks changed randomly across trials (DT-random condition). DeJong (1995) has shown that coordination demands differ between the conditions because the same scheduling strategy can be implemented throughout a block in DT-fixed, whereas in DT-random, the task processes have to be rearranged whenever the presentation order changes. This results in higher demands on the coordination of the processing streams in DT-random compared to DT-fixed. In other words, the requirement for frequent rearrangement makes the coordination of task-order scheduling, task interruption, switching, and reinstatement more demanding. Accordingly, we expected dual-task-related areas, as identified by the cognitive subtraction method in DT-fixed, to be more strongly activated in DT-random. Such a result of converging evidence by the cognitive subtraction and the parametric manipulation method would greatly increase the credibility and validity of our final interpretation.

\section{RESULTS}

\section{Behavioral Data}

If not otherwise noted, in the following analyses, either an analysis of variance (ANOVA) with repeated measures or two-sample paired $t$ tests were used. Significance for $t$ tests was calculated two-tailed. All error analyses were performed with arcsin-transformed relative error frequencies.

Analyses of RTs revealed a typical PRP effect in the DT-fixed condition (Figure 2). That is, the RTs on the second task (RT2) increased with decreasing SOA $[F(2,20)=$ 29.49; $p<.001$ ], while the RTs on the first task (RT1) remained constant over the range of SOAs $[F(2,20)=$ $0.36 ; p>.05$ ], resulting in an interaction of the factors Response (first or second) and SOA $[F(2,20)=294.30$; $p<.001]$. According to prior research (e.g., Pashler, 1994), the PRP effect points to concurrent drawing of both tasks on a bottleneck representing a limited capacity mechanism in dual-task processing (cf. Figure 1).

Further comparisons showed that RTs (Figure 3, symbols) increased significantly from VIS to AUD $[t(10)=$ $10.83 ; p<.001]$, from AUD to DT-fixed $[t(10)=2.70$; $p<.05]$ and from DT-fixed to DT-random $[t(10)=8.09$; $p<.001]$. For these comparisons, only RT1, averaged across the auditory and visual task, was used for the dual-task conditions. RT2s were further prolonged as compared to the RT1s in DT-fixed $[t(10)=10.07$; $p<.001]$, as well as in DT-random $[t(10)=9.77$; $p<.001]$. These results indicated reliable dual-task costs in both dual-task conditions compared to the single-task conditions. An error analysis indicated that dual-taskrelated RT increases were not due to a speed-accuracy

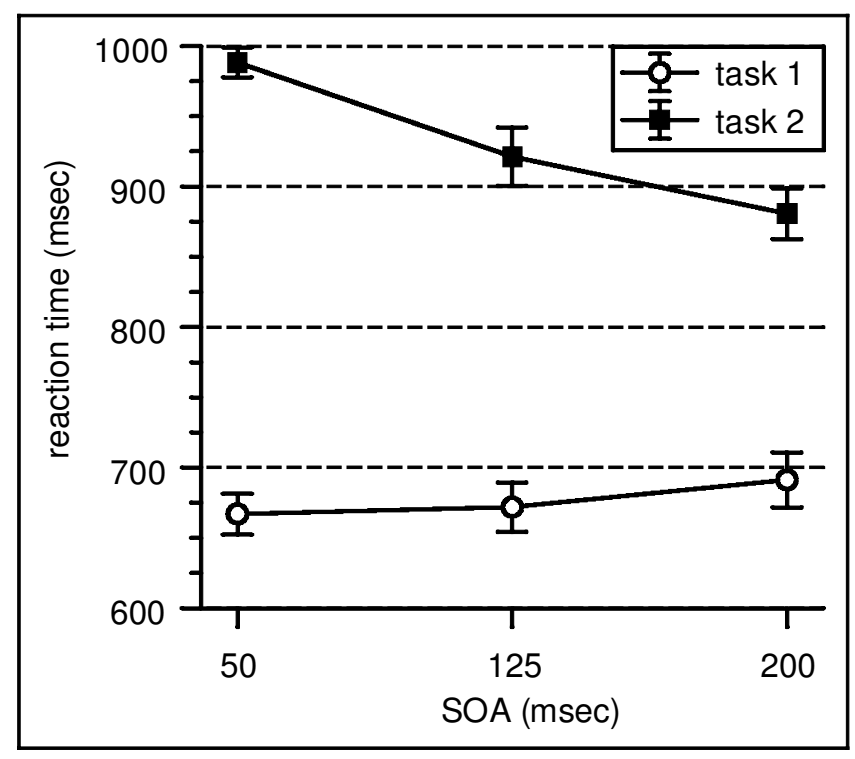

Figure 2. Behavioral data depicting the PRP effect. RTs plotted against the SOAs for DT-fixed. RTs are averaged across auditory and visual task. The RTs for the second task significantly increase with decreasing SOA, while the RTs of the first task are independent of the SOA. Error bars denote 95\% confidence intervals (Loftus \& Masson, 1994). 


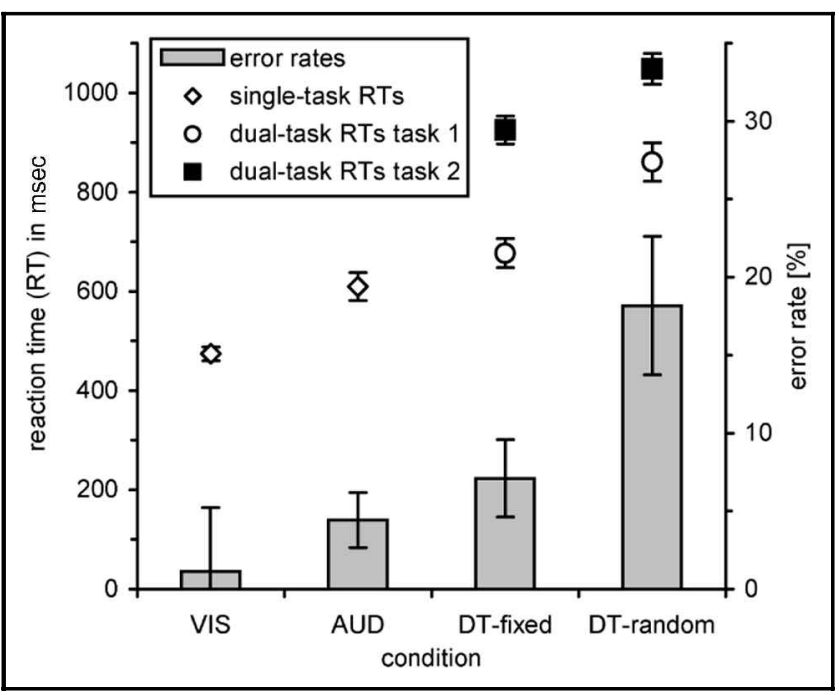

Figure 3. Behavioral data as a function of the different task conditions. Left axis and symbols denote RTs in msec; right axis and bars denote error rates, including reversal errors. For the dual-task conditions, RTs were averaged across the auditory and the visual tasks. Error bars denote 95\% confidence intervals (Loftus \& Masson, 1994).

trade-off. Participants made more errors (Figure 3, bars) in DT-random than in any other condition [smallest $t(10)=4.33$; all $p$ 's $<.01$ ], while they performed equivalently in all other conditions [highest $t(10)=2.02$; all $p$ 's $>.05]$. It has to be noted that dual-task trials, in which the correct keys were pressed according to the stimuli, but in the wrong order (reversals), were also scored as an error, and, consequently, they were included in the error analysis above. However, only $7.59 \%$ of the trials in the DT-random condition were reversal errors, which indicates that the participants were able to process the tasks in the correct order in this condition.

\section{fMRI Data}

\section{Single Tasks}

Activation in the single tasks was determined by comparing the functional data of these conditions with the resting baseline (AUD-BASE and VIS-BASE). In the following, we report prefrontal activations only. A complete list of significantly activated areas is given in Table 1. Both conditions led to rather small and circumscribed activation foci in the lateral prefrontal cortex (Figure 4c). In more detail, the analysis of the AUD condition revealed bilateral activation in the anteriorsuperior portion of the middle frontal gyrus (MFG; Brodmann's area [BA] 8, 9), while the analysis of the VIS condition revealed activation in anterior parts of the left MFG (BA 9) and right superior frontal gyrus (SFG; BA 8).

\section{Dual-Task Fixed Order}

The first goal of the present study was to examine dualtask-related activation by using the method of cognitive subtraction. For this purpose, we conducted an interaction analysis, composed of the following contrast: ((DT-fixed-AUD)-(VIS-BASE)). ${ }^{2}$ This comparison (Figure 4b, upper row; Table 2) revealed a large left hemispheric prefrontal activation in cortical areas lining the inferior frontal sulcus (IFS; BA 10, 45, 46), the MFG (BA 8, 9, 46), and the superior frontal sulcus (SFS; BA 6). The activation extended in the anterior-posterior axis from frontopolar regions to the precentral sulcus (PCS) and in the superior-inferior axis from the SFS to the IFS. This activation consisted of a chain of six interconnected (local) peaks along the anterior-posterior axis, with the highest peak located in the MFG (see Table 2, local peaks in italics). In the right hemisphere, cortical areas were activated in the mid portion of the MFG (BA 46) and in a region along the SFS (BA 6). These results show that performance of the DT-fixed condition led to extended bilateral prefrontal activation, which cannot be reduced to the summed effects of single-task performance. Further activation related to the dual-task processing was found along the intraparietal sulcus (IPS; BA 7) bilaterally, the left precuneus (BA $7 \mathrm{~m}$ ), the left middle temporal gyrus (BA 37) and the left cerebellum.

In the next step of the analysis, we tested whether the prefrontal activation in the DT-fixed condition was differentially located compared to the single-task conditions. For this purpose, we compared the locations of the peak activation (i.e., Talairach coordinates) in the left and right MFG in DT-fixed with the corresponding locations of the peak activation in the single tasks (left and right MFG in AUD, and left MFG and right SFG in $\mathrm{VIS}^{3}$, respectively). With respect to the statistical parametric maps (SPMs) of the group analysis, the activation peaks in the single-task conditions and dual-task conditions were separated by $17-30 \mathrm{~mm}$ (average $24 \mathrm{~mm}$; Euclidian distances). This indication of spatially different activation peaks was statistically confirmed by calculating the Euclidian distances for each subject individually. This analysis revealed Euclidian distances between 22 and $32 \mathrm{~mm}$ (average $27 \mathrm{~mm}$ ), which differed significantly from zero (all p's < .001; one-sample $t$ tests). Further analysis showed that the activation peaks in the singletask conditions were located more anteriorly ( $y$-axis according to Talairach coordinates, average $19 \mathrm{~mm}$ ) and medially ( $x$-axis, average $12 \mathrm{~mm}$ ) than the activation peaks in the DT-fixed condition (all p's $<.01$; two-sample $t$ tests). This indicates that single- and dualtask performances recruited different regions of the lateral prefrontal cortex.

\section{Dual-Task Random Order}

The second aim of the present study was to test whether the results revealed by the cognitive subtraction method could be validated by using a parametric manipulation method. For this purpose, we introduced 
Table 1. Stereotactic Coordinates (Talairach \& Tournoux, 1988) and Anatomical Location of (Local) Peak Activation in the Single-Task Conditions Compared with the Resting Baseline (AUD-BASE and VIS-BASE)

\begin{tabular}{|c|c|c|c|c|c|c|c|}
\hline \multirow[b]{2}{*}{ Anatomical Area } & \multirow[b]{2}{*}{$H$} & \multicolumn{3}{|c|}{$A U D$} & \multicolumn{3}{|c|}{ VIS } \\
\hline & & $B A$ & $(x, y, z)$ & $z$ & $B A$ & $(x, y, z)$ & $z$ \\
\hline \multicolumn{8}{|l|}{ Frontal } \\
\hline Middle frontal $G$ & $\mathrm{R}$ & $8 / 9$ & $28,42,32$ & 7.30 & & & \\
\hline Middle frontal $G$ & $\mathrm{~L}$ & $8 / 9$ & $-32,40,32$ & 7.40 & 9 & $-26,32,29$ & 5.18 \\
\hline Sup frontal $G$ & $\mathrm{R}$ & & & & 8 & $13,41,42$ & $4.25 *$ \\
\hline Medial frontal G & $\mathrm{R} / \mathrm{L}$ & 6 & $1,5,56$ & 10.61 & 6 & $-7,-10,56$ & 9.6 \\
\hline Precentral S & $\mathrm{R}$ & & & & $4 / 6$ & $37,-4,52$ & 7.4 \\
\hline Precentral S & $\mathrm{R}$ & $9 / 6$ & $46,4,41$ & 7.65 & $6 / 9$ & $46,4,41$ & 7.68 \\
\hline Precentral S & $\mathrm{L}$ & 6 & $-47,2,32$ & 7.82 & 6 & $-47,2,32$ & 5.73 \\
\hline Precentral G & $\mathrm{L}$ & $4 / 6$ & $-31,-10,53$ & 10.89 & $4 / 6$ & $-31,-10,56$ & 11.51 \\
\hline Central S & $\mathrm{R}$ & 4 & $34,-21,59$ & 10.99 & & & \\
\hline Central S & $\mathrm{L}$ & & & & 4 & $-35,-25,56$ & 12.61 \\
\hline Lateral S/insula & $\mathrm{L}$ & 45 & $-26,23,13$ & 5.60 & $40 / 42$ & $-38,-3,15$ & 5.6 \\
\hline Lateral S/insula & $\mathrm{R}$ & 44 & $43,12,6$ & 7.63 & 44 & $43,12,6$ & 5.21 \\
\hline \multicolumn{8}{|l|}{ Parietal } \\
\hline Inf parietal lobe (IPS) & $\mathrm{R}$ & 40 & $47,-41,39$ & 9.00 & $22 / 40$ & $50,-44,27$ & 6.92 \\
\hline Inf parietal lobe (IPS) & $\mathrm{L}$ & 40 & $-47,-42,36$ & 9.74 & 41 & $-53,-26,23$ & 8.3 \\
\hline Sup parietal lobe & $\mathrm{R}$ & 7 & $11,-64,59$ & 6.10 & 5 & $34,-46,58$ & 5.64 \\
\hline Sup parietal lobe (precuneus) & $\mathrm{L}$ & 7 & $-13,-66,53$ & 5.76 & 7 & $-16,-70,60$ & $4.41 *$ \\
\hline
\end{tabular}

\section{Temporal}

Sup temporal G/lateral S

Sup temporal G/lateral S

$\begin{array}{lllr}\mathrm{L} & 41 & -44,-26,11 & 13.25 \\ \mathrm{R} & 41 / 42 & 53,-30,18 & 13.38 \\ \mathrm{~L} & 37 / 39 & -47,-58,18 & 7.76\end{array}$

Sup temporal S

$-47,-58,18$

7.76

\section{Other}

Calcarine S

L 17

Occipital G

R 18

$-16,-89,4$

6.32

17

$-20,-89,5$

7.96

Globus pallidus

L

14, $-94,8$

7.60

17

$-20,-89,4$

Globus pallidus

$-13,0,5$

5.01

Thalamus

R

$20,-4,10$

$4.74 *$

Thalamus

R

10, -16, 12

5.85

Cerebellum

L

$-11,-17,9$

$4.14^{*}$

$-14,-21,16$

$-14,-55,-9$

9.46

Cerebellum

$26,-67,-9$

6.04

$23,-52,-12$

8.74

Activation is thresholded at $z>3.3$ ( $p<.0005$, uncorrected).

Abbreviations: $\mathrm{H}=$ Hemisphere $(\mathrm{L}=$ left, $\mathrm{R}=$ right $)$; $\mathrm{BA}=$ Brodmann's area; $\mathrm{G}=$ gyrus; $\mathrm{S}=$ sulcus; Sup = superior; Inf $=$ inferior; Ant $=$ anterior; IPS = intraparietal sulcus; IFS $=$ inferior frontal sulcus; MFG $=$ middle frontal gyrus.

Statistical significance according to Bonferroni adjustment: An asterisk denotes activation peaks, which proved nonsignificant after Bonferroni correction for multiple comparisons. Significance levels after Bonferroni correction: $p<.05$ corresponds to $z>4.79 p<.01$ to $z>5.11$, and $p<.0001$ to $z>5.92$. 
Table 2. Stereotactic Coordinates (Talairach \& Tournoux, 1988) and Anatomical Location of (Local) Peak Activations in the Dual-Task Conditions

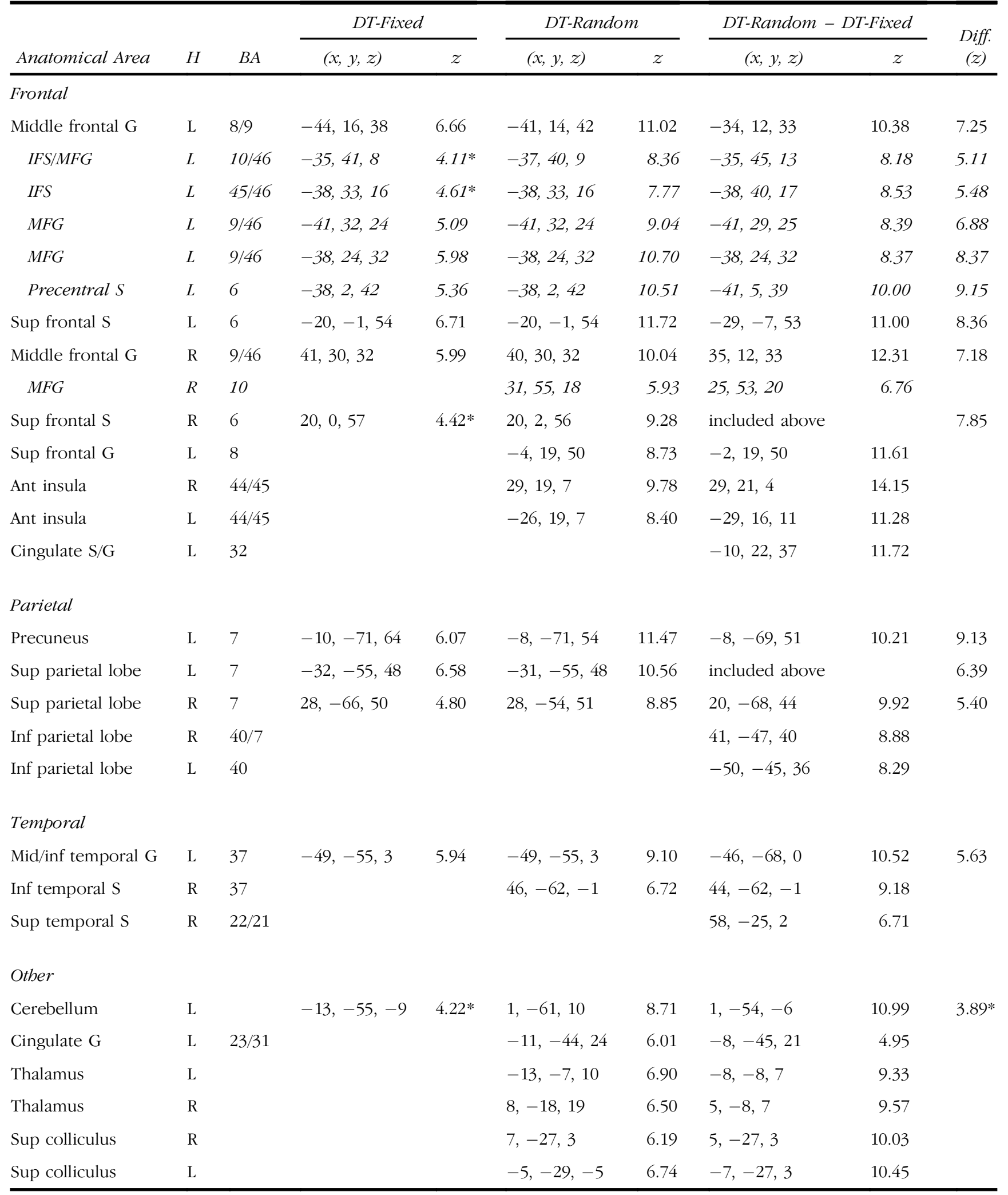

DT-fixed and DT-random denote the results of the interaction analyses; DT-random-DT-fixed denotes the results of the direct comparison of both conditions. Diff. depicts the $z$ value in the SPM of the comparison DT-random-DT-fixed at the location of the peak activation as defined in the interaction analysis of DT-fixed. Local activation peaks of frontal areas are printed in italics.

Statistical significance according to Bonferroni adjustment: An asterisk denotes activation peaks, which proved nonsignificant after Bonferroni correction for multiple comparisons. Significance levels after Bonferroni correction: $p<.05$ corresponds to $z>4.79, p<.01$ to $z>5.11$, and $p<.0001$ to $z>5.92$. For further information, see Table 1 . 

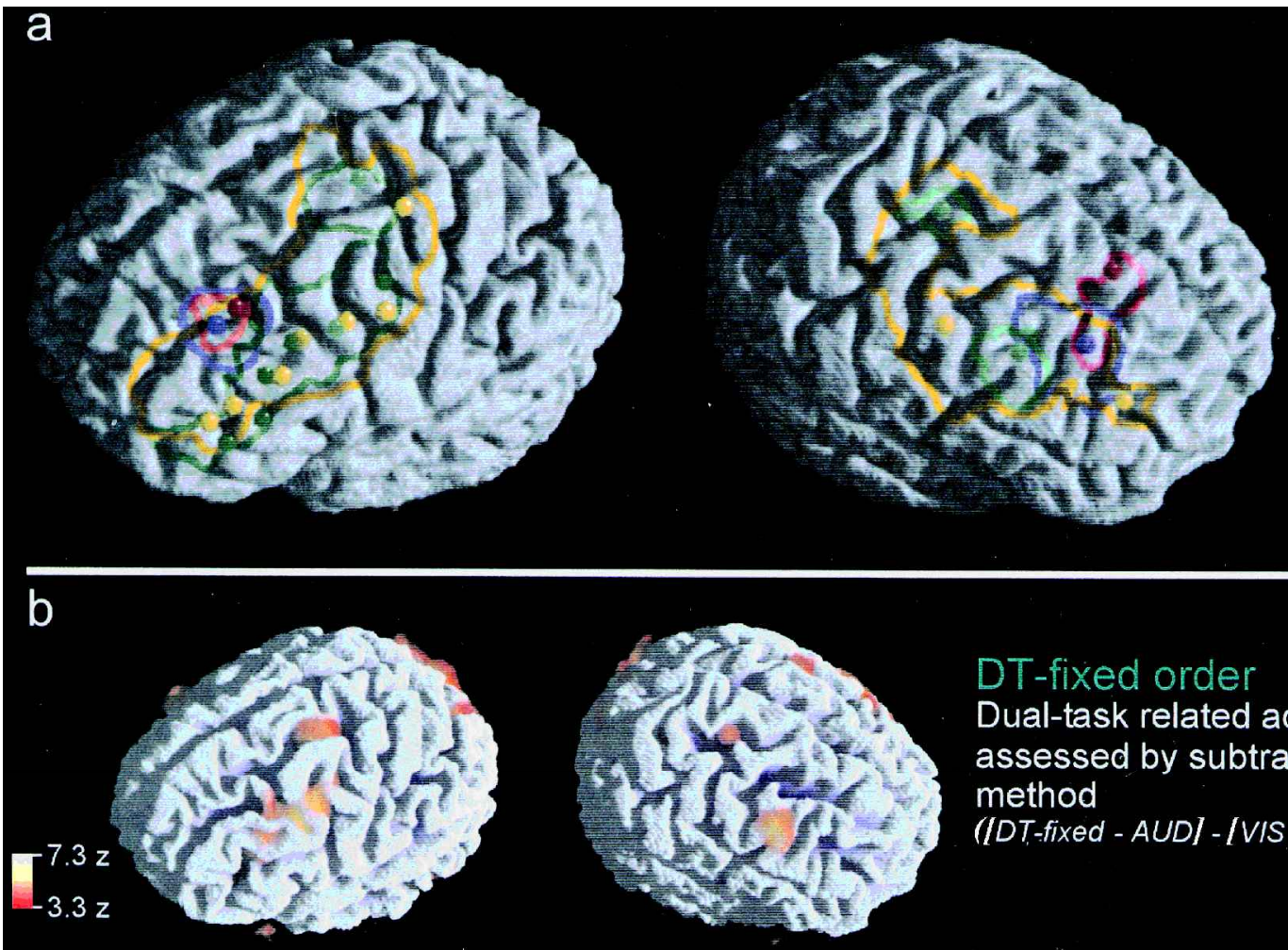

DT-fixed order

Dual-task related activation assessed by subtraction method

(IDT,-fixed - AUDI - IVIS - BASEI)
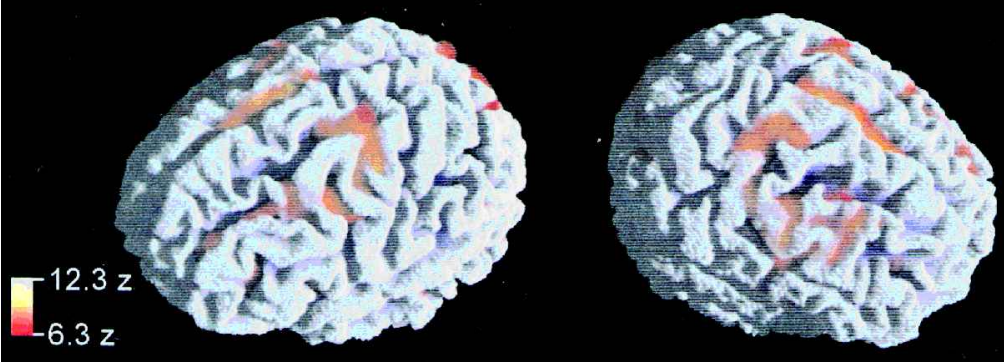

DT-random order Dual-task related activation assessed by parametric manipulation method (DT-random - DT-fixed)

C
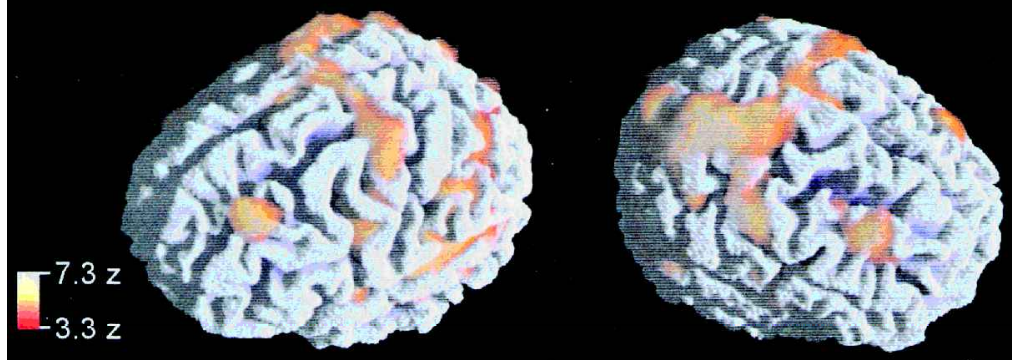

Auditory single task (AUD - BASE)
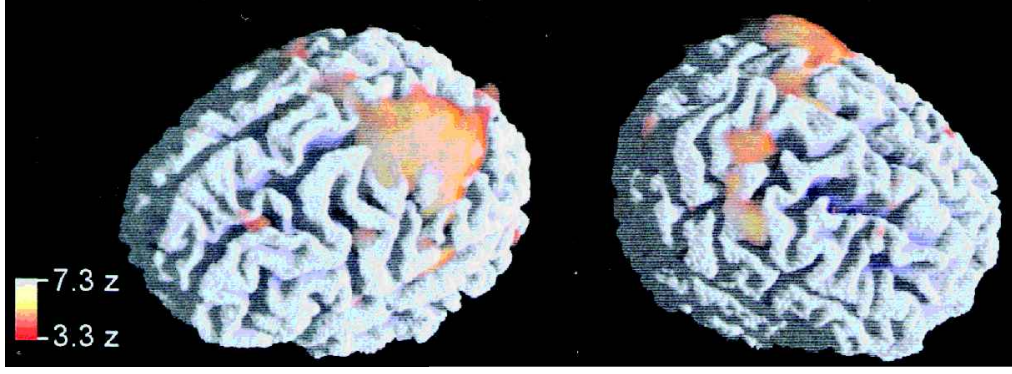

Visual single task

(VIS - BASE) 
the DT-random condition and predicted that dual-taskrelated areas as defined by the DT-fixed condition should reveal higher activation in the DT-random condition. To ensure that DT-random activated similar areas as DT-fixed, we first calculated the same interaction contrast as for DT-fixed. Then, we compared the activation in both tasks directly. Again, lateral prefrontal activations are reported only, and a complete list of activated areas is given in Table 2 .

First, the results of the subtraction analysis of the DT-random condition showed activation in similar areas as in the DT-fixed condition. In the left hemisphere, a widely spread activation, covering the IFS (BA 10, 45, 46), the MFG (BA 8, 9, 46), and the SFS (BA 6), was found. In the right hemisphere, cortical areas in the MFG (BA 10, 46) and regions along the SFS (BA 6) revealed significant activation. The activation in the MFG (especially in the right hemisphere) extended more anteriorly in the DT-random condition than in DT-fixed. However, most importantly, the (local) activation peaks were nearly identically localized compared to the DT-fixed subtraction analysis. This was also true if more conservative $z$ thresholds were used for the statistical analysis (Table 2). To summarize, this analysis indicated almost identical anatomical structures in the lateral prefrontal cortex to be involved in the processing of DT-fixed and DT-random.

Second, we calculated the contrast between DT-random and DT-fixed in order to obtain a direct comparison between both dual-task conditions. The results of this analysis revealed higher activation in the DT-random condition in all cortical areas activated in the DT-fixed condition (Figure 4b, lower row; Table 2). Specifically, the lateral prefrontal cortex was extensively activated bilaterally (covering the IFS, MFG, and SFS).

To exclude the possibility that the stronger activation in DT-random is due to the increased error rate in this condition, we calculated the same contrasts using only completely error-free blocks. This analysis (not shown) revealed an almost identical pattern for the relevant activation foci, which indicates that the observed dualtask-related activation was not caused by increased error processing in the DT-random compared to the DT-fixed condition.

Taken together, these results show dual-task-related activation in DT-fixed, which cannot be reduced to the summed single-task activation. Furthermore, although prefrontal activation was also observed in the single tasks, this activation was differentially located. All dual-taskrelated areas as defined by DT-fixed, especially lateral prefrontal areas, showed higher activation in DT-random, with striking similarities regarding the location of activation peaks. Therefore, dual-task-related activation was shown with two different methodological approaches, first, with an analysis based on the subtraction method, and, second, with the parametric manipulation method.

\section{Regions-of-Interest (ROI) Analyses}

To assess the effects of the different task conditions on the strength of activation in lateral prefrontal areas in more detail, we conducted ROI analyses. As described in the Methods, these analyses are based on an analysis of a cloud of voxels, surrounding the voxel with peak activation in dual-task-related foci of activation. As reference points, we determined the voxel that was located at $x=-44, y=16, z=38$ for the left lateral prefrontal cortex and the voxel at $x=41, y=30, z=32$ for the right lateral prefrontal cortex (cf. Table 2). In the first step of this analysis, we tested whether the percent signal change (PSC; see Methods) in the conditions VIS, AUD, DT-fixed, and DT-random differed significantly from that in the condition BASE (Figure 5). While the PSC in the single tasks did not differ significantly from BASE (lowest $p>$.19), it differed in the dual-task conditions (highest $p<.05$ ). In a next step, we tested whether the PSC in the DT-fixed condition differed from the PSC in each single-task condition. This analysis revealed a significantly higher PSC in DT-fixed than in each single task (all $p$ 's $<.05$ ). Furthermore, DT-random showed a higher PSC than DT-fixed (all p's < .01). Altogether, these results show that there is only a minor, nonsignificant signal change in the single-task conditions in areas identified as dual-task related by means of the SPMs. Furthermore, the results show that dual-task performance in DT-fixed significantly enhances the BOLD-signal in these areas and that this enhancement is more pronounced in the more difficult DT-random condition.

Finally, we tested whether the PSC observed in the dual-task conditions can be reduced to the sum of the PSC observed in the single-task conditions. For this purpose, we compared the PSC in each dual-task condition with the summed PSC of both single-task conditions for each ROI. The analyses showed that the PSC increased overadditively in DT-fixed, as well as in DT-random, as compared to the summed PSC of the single-task conditions (all p's < .05). In other words, the observed PSC in the dual-task conditions were not reducible to the summed effects of the single-task

\footnotetext{
Figure 4. Averaged fMRI data of 11 participants. Note the different thresholds of the SPMs, which were selected for purpose of illustration. (a) Contours and (local) peaks of the activated areas projected onto the surface of a reference brain, for the thresholds as given in b and c. Colors correspond to the condition denotation in b and c. Note that contours might change with different thresholds. (b) Activation is combined with the same, but white matter segmented, reference brain. Activation inside white matter is not shown. Both cognitive subtraction approach (upper row) and parametric manipulation approach (lower row) revealed dual-task-related activation in lateral prefrontal cortices. (c) Activation related to single-task performance.
} 
Figure 5. Average PSC for ROIs in the left and right middle frontal gyrus. PSC in the dual tasks was higher than the summed PSC of both single tasks. PSC in DT-random was higher than in DT-fixed.

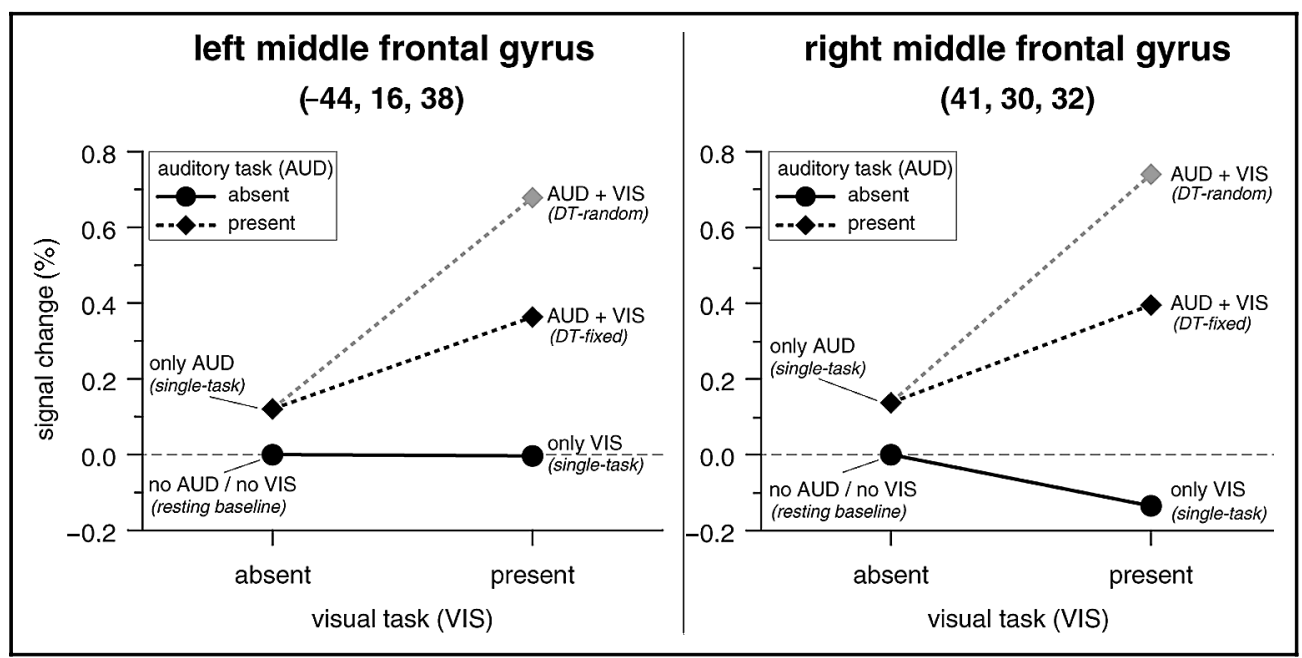

conditions. Therefore, this analysis confirmed the results observed in the SPMs.

\section{DISCUSSION}

The concurrent performance of two choice-reaction tasks resulted in additional activation in the dorsolateral prefrontal cortex (DLPFC; i.e., IFS/MFG) as compared to the single-task performance. This activation was accompanied by severe performance decrements in the dualtask compared to the single-task situations. Furthermore, the finding of dual-task-related activation was validated by converging evidence with two methodologically different neuroimaging approaches: the cognitive subtraction method and the parametric manipulation method.

The results suggest that by using a dual-task paradigm that ensures overlapping and interfering task processing, cortical areas mediating the coordination of task processing can be localized. Thus, the identified areas along the IFS and in the MFG seem to be involved in the fast adaptation and coordination of actions according to current behavioral goals, especially in situations of interfering information.

\section{Dual-Task-Related Brain Areas Assessed by the Subtraction Method}

In a first step, we identified dual-task-related brain areas in the DLPFC with the cognitive subtraction method. For this purpose, participants performed a dual task according to the PRP paradigm and the corresponding component tasks as single tasks. The comparison of dual-task and single-task activation revealed activation not reducible to the summed effects of the activation in both single tasks performed separately. Such activation was located in lateral prefrontal areas along the IFS and in the MFG. The finding of additional dual-task-related activation indicates that certain processing requirements must have been additionally present in the dual-task compared to the single-task situation.
We propose that these additional requirements reflect executive functions coordinating interfering processes of the component tasks. In the present DT-fixed condition, the occurrence of dual-task interference was indicated by the PRP effect, that is, the increase of RTs on the second of two consecutively presented tasks (Pashler, 1994). Recent dual-task theories suggest that the resolution of this interference requires executive functions, which schedule the processing order of the tasks, interrupt and reinstate task processing, and switch between the processing streams of the tasks (Meyer \& Kieras, 1997; DeJong, 1995). Our data indicate that these processes are mediated by lateral prefrontal areas along the IFS and in the MFG. Furthermore, they show that even two easy-to-perform, nonmnemonic tasks can impose substantial coordination demands on these areas when they are performed concurrently.

The present findings converge with those of other neuroimaging studies investigating related processes with different paradigms. In particular, it has been shown that the inhibition of a task (Konishi et al., 1999), the switching between tasks (Dove, Pollmann, Schubert, Wiggins, \& von Cramon, 2000; Sohn, Ursu, Anderson, Stenger, \& Carter, 2000) and the instatement of nondominant tasks (Zysset, Müller, Lohmann, \& von Cramon, 2001) also correlate with activation in cortical areas along the IFS and in the MFG. Accordingly, these and our results suggest that these cortical areas implement general control over task coordination, regardless of the particular paradigm used, as long as interfering information has to be processed (see also MacDonald, Cohen, Stenger, \& Carter, 2000; Miller, 2000).

The present findings are further supported by the results of Koechlin et al. (1999), Goldberg et al. (1998), and D'Esposito et al. (1995), who also showed that performance of a dual task relies on additional neural processing in cortical areas of the MFG, as compared to single-task performance. Moreover, a recent study by Herath et al. (2001) identified additional activation in a variant of the PRP paradigm, too. However, in this study, 
dual-task-related activation was identified in the right inferior frontal gyrus (IFG). The reason for this difference is not completely clear but might result from differences between the employed component tasks. Herath et al. used simple-reaction tasks (visual and somatosensory), while we used choice-reaction tasks (visual and auditory). Beside the combination of different modalities, processing differences between simple- and choice-reaction tasks (Schubert, 1999; Pashler, 1994; Frith \& Done, 1986) might have resulted in the involvement of different cortical areas in the Herath et al. and the present study.

The results of several other dual task studies are not in accordance with our findings. In particular, Adcock et al. (2000), Bunge et al. (2000), and Klingberg (1998) failed to find any additional prefrontal areas associated with dualtask performance. There may be several reasons for the differences between these findings and ours. For example, these studies used rather complex paradigms, which did not allow the occurrence of interference to be controlled. Due to rather long intervals between the component tasks, the tasks may have been processed without simultaneously drawing on a bottleneck mechanism. Consequently, interference and executive functions would have been absent and the brain areas related to these functions could not be localized. This interpretation is supported by the fact that Adcock et al. and Bunge et al. reported only marginal and even no performance decrements, respectively, in the dual-task conditions. This lack of dual-task decrements would have been counterintuitive if interference between both tasks had been present in these studies. Such caveats were circumvented in the present study by using the PRP paradigm with short SOAs between both component tasks.

A further reason for the failure to detect dual-taskrelated activation might be the type of single tasks employed. For instance, Adcock et al. used a semantic categorization and a mental rotation task, and Bunge et al. a sentence evaluation and a memory task, while we used easy-to-perform choice-reaction tasks. It is conceivable that in the former studies, even the single tasks alone involved executive functions. Accordingly, in the abovementioned studies, prefrontal activation was already present during single-task performance in inferior regions of the DLPFC (IFS/ventral MFG), that is, those areas that proved to be dual-task related in the present study. Thus, if dual-task performance relies on these more inferior regions of the DLPFC, one should expect overadditively stronger activations in areas already activated by single-task performance, rather than newly activated areas. The results of Adcock et al. (2000), Bunge et al. (2000), and Klingberg (1998) support this interpretation because these studies showed trends of higher activation in the MFG during dual-task compared to single-task performance.

In the present study, the performance of the single tasks also resulted in activation of lateral prefrontal areas. However, the foci of these activations were located in anterior-superior portions of the MFG and SFG, and, therefore, were differentially located compared to the dual-task-related activation.

Anterior-superior portions of the PFC are assumed to receive input from higher-order auditory and visual association areas (Miller, 2000; Petrides \& Pandya, 1999; Romanski et al., 1999). In the present study, activation strength in these areas remained constant during all task conditions. Consequently, it was subtracted out in the dual-task interaction contrasts. This would be in accordance with the view that activation of these prefrontal regions purely reflects the input of domain specific sensory information into the prefrontal cortex, while the cortical areas identified as dual-task related reflect executive functions acting upon this information to resolve interference. This is in disagreement with a suggestion made by Adcock et al. (2000) and Bunge et al. (2000) that additional processing in dual-task performance is solely mediated by an increase of activation in brain areas directly related to single-task processing. The ROI analyses of the present data showed that there was no activation in dual-task-related areas during single-task performance. Furthermore, the voxels of peak activation in the dual-task and the singletask conditions were clearly spatially separated. These results suggest that executive functions resolving interference in dual-task processing can be attributed to specific cortical areas and that these functions do not solely originate from altered processing in areas subserving component-task processing, as proposed by Adcock et al. and Bunge et al.

In the present study, performance of the dual task activated not only prefrontal cortices, but also parietal areas. These activations were located mainly along the IPS and the precuneus. These parietal activations are in accordance with other paradigms that also investigated executive functions (Koechlin et al., 1999; Rowe, Toni, Josephs, Frackowiak, \& Passingham, 2000; Callicott et al., 1999; Schubert, von Cramon, Niendorf, Pollmann, \& Bublak, 1998; Cohen et al., 1997), suggesting that executive functions might be mediated by a network of brain areas including prefrontal and parietal cortices (Bunge et al., 2000; Baddeley \& Della Sala, 1996; Baddeley, 1998). In more detail, the presently observed areas along the IPS were previously associated with more general attentional functions, like different types of visual attention (Wojciulik \& Kanwisher, 1999) or the attention to time intervals (Coull \& Nobre, 1998). Such attentional demands specifically arise in the dual-task situation, where attention has to be switched rapidly between modalities in a predefined order.

\section{Dual-Task-Related Brain Areas Assessed by Parametric Manipulation}

As mentioned in the Introduction, the approach of cognitive subtraction is based on the critical presumption of 
pure insertion (Donders, 1868/1969), that is, that processing of the auditory and visual task is the same, regardless of whether they are performed as single tasks or as component tasks in a dual-task context. Furthermore, in dual-task blocks, two task sets have to be held in memory, compared with one task set in single-task blocks (Kray \& Lindenberger, 2000; see also Dove et al., 2000; Klingberg, 1998). The increased memory load might interact with task-dependent processes, resulting in the overadditive activation observed in the DT-fixed condition (Rypma, Prabhakaran, Desmond, Glover, \& Gabrieli, 1999).

To circumvent possible pitfalls of pure insertion and memory load, we additionally used the parametric manipulation method to assess dual-task-related activation. For this purpose, we manipulated the difficulty of dual-task-specific executive functions by introducing the DT-random condition. Compared to DT-fixed, this second dual-task condition imposed higher demands on executive functions, which coordinate interfering task processing at the stage of the bottleneck (DeJong, 1995). The behavioral data showed that DT-random was considerably more demanding than DT-fixed. The direct comparison of the fMRI data revealed that both conditions engaged nearly identical anatomical structures with remarkable similarities with respect to the location of the (local) activation peaks. Most importantly, these anatomical structures were stronger activated by means of $z$ value and PSC in the more demanding DT-random compared to the DT-fixed condition.

These data validate the conclusion suggested by the results of the cognitive subtraction method, that the coordination of interfering task processes activates additional cortical areas in dual-task compared to single-task situations. In addition, the validity of the parametric manipulation approach is emphasized by the fact that cortical areas related to single-task processing, like anterior-superior portions of the DLPFC or primary sensory and motor cortices, showed virtually no increase in activation strength in DT-random compared to DT-fixed (cf. Cohen et al., 1997). This rules out an alternative suggestion that early perceptual or late motor processes were affected by the parametric manipulation of dual-task difficulty and not the proposed coordination of interfering processing stages at the bottleneck. As outlined above, this coordination might involve the scheduling of the processing order, as well as the management of task interruption and the switching between different processing streams (Meyer \& Kieras, 1997; DeJong, 1995). It is still an open issue whether these aspects are realized by distinct subprocesses or whether they rely on the same cognitive mechanism. It would be an interesting question for future studies to elucidate whether a parametric manipulation of the dual-task difficulty different than the one employed in our study would lead to differently localized activation changes in the observed dual-task-related areas.
In addition to similarly localized activation foci, there were also slight differences between the activation patterns observed in the DT-fixed and DT-random conditions, for example, in the medial SFG. Such differences might result from certain cognitive requirements in the DT-random condition, which are related to side aspects of the parametric manipulation. For instance, the observed DT-random activation in the medial SFG might be due to the additional requirement to perceive the presentation order of the stimuli or to the requirement to change rapidly the order of motor programs in DT-random, which were present only in a very rudimentary form in DT-fixed. This would be in accordance with studies indicating a specific role of this area in the perception of stimulus sequences (Schubotz, Friederici, \& von Cramon, 2000; Schubotz \& von Cramon, 2001) or the programming of motor sequences (Shima \& Tanji, 1998, 2000).

\section{Conclusion}

Taking together the findings gained by the cognitive subtraction and parametric manipulation method, we presented strong evidence that lateral prefrontal areas along the IFS and in the MFG are associated with dualtask performance. More specifically, these areas proved to be related to the coordination of interfering task processing, and not to memory load or altered componenttask processing. Such coordination requires the rapid adaptation of current processing strategies and involves the inhibition and activation of task representations and the switching between them (cf. Baddeley, 1986). While this adaptive processing was already required by the bottleneck processing in the DT-fixed condition, it played an even greater role in the DT-random condition. Here, processing strategies for handling the interference caused by the bottleneck had to be rapidly changed and adapted on-line during task presentation. Interestingly, even the merging of two rather easy-to-perform tasks can impose considerable demands on these coordination processes, as indicated by the substantial activations and severe performance decrements in the dual-task conditions. Therefore, we conclude that cortical areas along the IFS and in the MFG mediate the rapid adaptation of processing strategies, which is a basic prerequisite for goal-oriented and coherent behavior in situations where multiple conflicting actions have to be performed in a defined manner.

\section{METHODS}

\section{Participants}

Twelve participants took part in the fMRI experiment, each having given prior informed consent according to the Max-Planck-Institute guidelines. The study was approved by the local ethics review board at the 
University of Leipzig, Germany. All participants were right handed as assessed by the Edinburgh Inventory (Oldfield, 1971). Due to technical problems, the data of one participant had to be discarded, resulting in 11 participants for the analyses. The age of the remaining 11 participants (5 women) ranged from 21 to 27 years, with an average of 24 years. All participants had normal or corrected to normal vision.

\section{Stimuli and Procedure}

Participants had to perform an auditory and a visual three-choice reaction task. These tasks were performed blockwise either alone (single-task conditions) or together (dual-task conditions). While lying in the fMRI scanner, participants viewed a projection screen via a mirror. They responded on two separate fMRI-suitable keypads, each with four keys. The tasks were as follows (see Figure 6).

\section{Single Tasks}

Visual single task (VIS). A trial in the VIS condition started with a blank green screen for $150 \mathrm{msec}$, followed by a fixation period of $850 \mathrm{msec}$. During this fixation period, three black squares (each $1.6^{\circ} \times 1.6^{\circ}$ ) were presented on a green background, and the middle square contained a fixation cross $\left(0.38^{\circ} \times 0.38^{\circ}\right)$. The middle square was located at the center of the screen and the two other squares horizontally to the left and right, each with a gap of $0.44^{\circ}$. After the fixation period, one of the three squares (the target) changed its luminance from black to a light gray for $300 \mathrm{msec}$, while the other two black squares remained on the screen. After the presentation of the target stimulus, the screen was cleared and the participants had to respond during an interval of $1750 \mathrm{msec}$. Participants had to respond with the right index finger to the left, with the right middle finger to the middle and with the right ring finger to the presentation of the right target square. After responding, either a blank screen or a visual error feedback was presented for $250 \mathrm{msec}$.

Auditory single task (AUD). A trial in the AUD condition started with the identical blank screen and fixation period as the visual single task. After the fixation period, a tone with a frequency of either 300,600 , or $1300 \mathrm{~Hz}$ was presented for $300 \mathrm{msec}$, while three black squares were presented on the screen. After the presentation of the tone, the screen was cleared. The participants had to respond to the low tone with their left ring finger, to the middle tone with the left middle finger, and to the high tone with the left index finger. The other characteristics of the procedure were identical to the condition VIS.

\section{Dual Tasks}

In the dual-task conditions, the participants had to perform both tasks together. For this purpose, both stimuli (auditory and visual) were presented in rapid succession, separated by the SOA. Participants were instructed to respond in the order of task presentation, that is, stimulus presentation. The order of task presentation was balanced, so that an equal number of trials started with the auditory and visual stimulus, respectively. There were two dual-task conditions: DT-fixed and DT-random.

DT-fixed. In this condition, the order of task presentation remained constant within a block. Participants were informed about the upcoming order (AUD-VIS
Figure 6. Trial design. The time courses are shown on the left, the stimulus-response mappings on the right. In the dual task, the participants had to combine both mappings. The gray bar denotes the presentation time of the auditory stimulus. Each trial lasted 3300 msec. (a) Visual single task. (b) Auditory single task. (c) Dual task. In this example, the participants first had to respond to the auditory and then to the visual stimulus. In DT-random, the SOA was $200 \mathrm{msec}$.

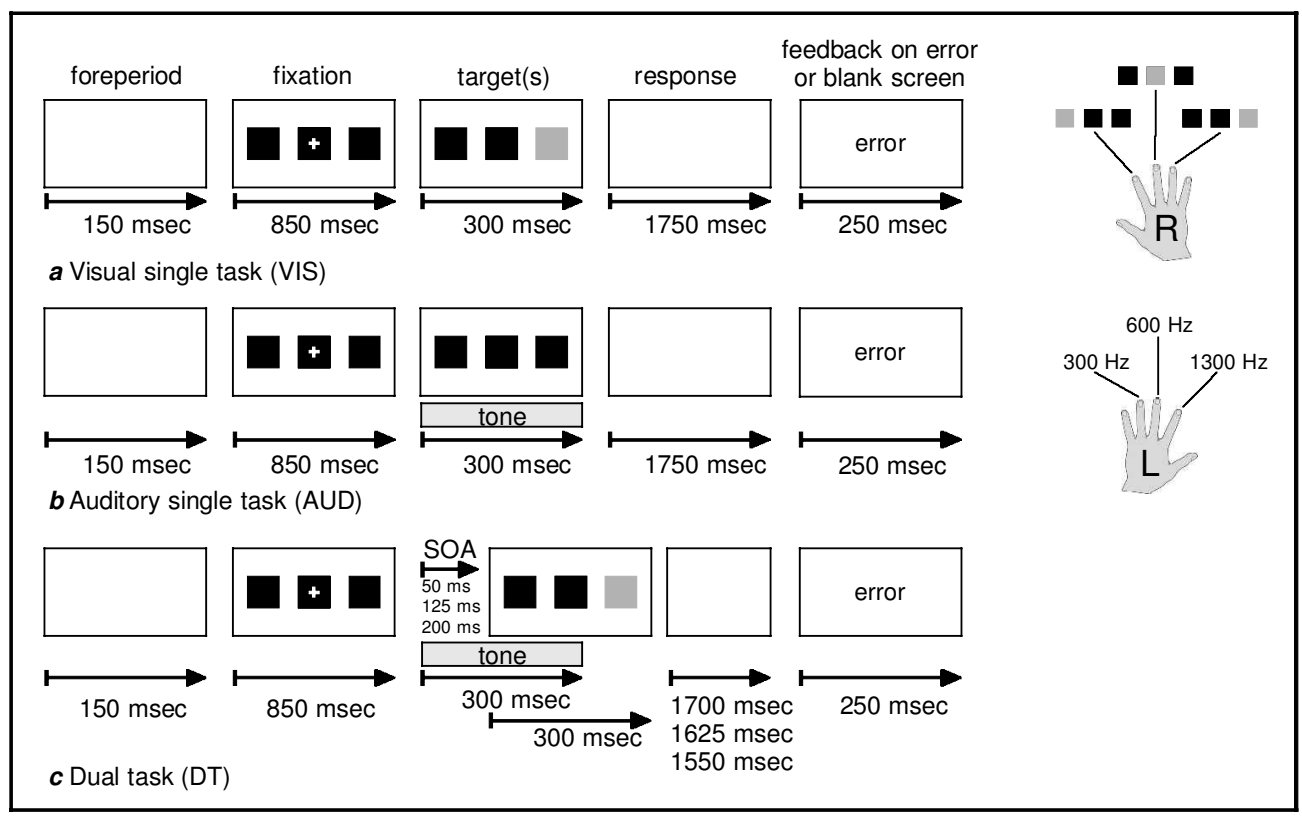


or VIS-AUD) by an instruction given immediately before each block. The SOA varied randomly between 50, 125, and $200 \mathrm{msec}$. To ensure that the total trial duration was the same in every condition, the time available to respond to the second task was decreased by the amount of the used SOA, so that the available response time for the second task in the SOA 200 msec condition decreased to $1550 \mathrm{msec}$. The different SOAs were used to show the PRP effect as an indicator of concurrent task processing. For the analysis of the fMRI data, the SOAs were averaged. All other characteristics were identical to the single-task conditions.

DT-random. In this condition, the order of task presentation varied pseudorandomly across trials. Participants received no information about the upcoming stimulus order, but, instead, they had to perceive the presentation order and adapt their response accordingly. We used only one SOA of $200 \mathrm{msec}$. Prior testing revealed this SOA as most appropriate for convenient order detection by the participants.

BASE. Additionally, we included a resting baseline condition (BASE), in which the participants were required to fixate a black cross $\left(0.38^{\circ} \times 0.38^{\circ}\right)$, presented on a green background at the center of the screen.

Design of measurement. A block design was used, with each block consisting of nine trials, resulting in a duration of $29.7 \mathrm{sec}$ per block. The blocks where separated by an interblock interval of $10.3 \mathrm{~s}$, which also served as instruction period for the task in the following block. A session consisted of two runs, each beginning and ending with the condition BASE. Singleand dual-task conditions were counterbalanced, so that the probability of transitions between both conditions was equal, with the only exception that neither single-task nor dual-task conditions were presented in direct succession. All conditions were presented eight times, resulting in a total experimental runtime of $24 \mathrm{~min}$. All participants received an identical stimulus protocol. One to three days before the fMRI measurement, participants practiced the tasks outside the fMRI scanner.

\section{Scanning Procedure}

Imaging was carried out with a 3T scanner (Medspec 30/100, Bruker, Ettlingen, Germany) equipped with a standard birdcage head coil. Participants were supine on the scanner bed, and cushions were used to reduce head motion. Fourteen axial slices $(19.2 \mathrm{~cm}$ FOV, $64 \times$ 64 matrix, $5 \mathrm{~mm}$ thickness, $2 \mathrm{~mm}$ spacing), parallel to the $\mathrm{AC}-\mathrm{PC}$ plane and covering the whole brain were acquired using a single shot, gradient recalled EPI sequence (TR $2 \mathrm{~s}$, TE $30 \mathrm{msec}, 90^{\circ}$ flip angle) sensitive to BOLD contrast. Two functional runs with 360 volumes each were administered, with each volume sampling all 14 slices. Prior to the functional runs, 16 anatomical MDEFT slices and 16 EPI-T1 slices were acquired. In a separate session, high-resolution whole-brain images were acquired from each participant using a T1-weighted three-dimensional segmented MDEFT sequence. These images were linearly rotated and translated, but not resized, into the stereotactic space of Talairach and Tournoux (1988).

\section{Data Analysis}

Preprocessing

The fMRI data were analyzed using the software package LIPSIA (Lohmann et al., 2001). First, the functional data were preprocessed. For this purpose, artifacts at scan borders were removed and a slicewise movement correction in the transverse direction was applied (Friston, Williams, Howard, Frackowiak, \& Turner, 1996). A Gaussian spatial filter (FWHM $5.65 \mathrm{~mm}$ ) was used for smoothing. The temporal offset between acquisition times of different slices acquired in one volume were corrected using a linear interpolation.

After preprocessing, the functional and anatomical data were coregistered: First, the MDEFT and EPI-T1 slices geometrically aligned with the functional slices were coregistered with the high-resolution 3-D reference T1 data set of each participant. Rotational and translational parameters computed for this registration were stored in individual transformation matrices. Second, each transformation matrix was transformed into a standard brain size (Talairach \& Tournoux, 1988) by linear scaling. Finally, these normalized transformation matrices were applied to the individual fMRI data. After anatomical coregistration, the functional data were spatially rescaled to a resolution of $3 \mathrm{~mm}^{3}$ using trilinear interpolation.

\section{Statistics}

Statistical analysis was based on a voxelwise least squares estimation using the general linear model for serially autocorrelated observations (Friston et al., 1995). A boxcar function with a response delay of $6 \mathrm{sec}$ was used to generate the design matrix. Lowfrequency signal drifts were controlled by applying a temporal highpass filter with a cutoff frequency of $0.0036 \mathrm{~Hz}$. In addition, the design matrix and the functional data were linearly smoothed with a 4-sec FWHM Gaussian kernel. The emerging autocorrelation caused by the temporal filtering and the smoothing was taken into account during statistical evaluation by an adjustment of the degrees of freedom (Worsley \& Friston, 1995). Contrasts between different conditions were calculated using the $t$ statistics. Subsequently, $t$ values were transformed into $z$ scores. As the individual 
functional data sets were aligned to the same stereotactic reference space, a group analysis of fMRI data was performed using a voxelwise one-sample $t$ test (Bosch, 2000). All resulting SPMs were thresholded at $z>3.3$ $(p<.0005$, uncorrected). To account for the possibility of false positives (Type I errors) given by the number of multiple tests, we further performed a Bonferroni adjustment for an overall false-positive probability of .05 (corresponding $z>4.79$ ). The results of this adjustment are given in Tables 1 and 2 .

\section{ROI Analyses}

As a second approach for analyzing the data, we performed ROI analyses. These analyses were aimed at testing whether the effects observed in the SPMs were also observed in the PSC of the BOLD response. The analyses were performed as follows: First, we determined the activation peaks of dual-task-related activation in lateral prefrontal areas as revealed by the SPM of the DT-fixed interaction group analysis. Starting from these voxels, groups of continuously connected voxels exceeding a threshold of $z>5$ were determined. Next, the signal timecourses of the voxels constituting these groups were individually averaged for each condition. PSC of the AUD, VIS, DT-fixed, and DT-random conditions was individually calculated by relating their timecourses to the timecourse of the BASE condition. To avoid influences from transient adjustment processes at the beginning or at the end of a block, we discarded the first and last four timesteps from each timecourse, leaving seven timesteps for analysis. Next, the PSC of the seven timesteps was averaged, so that one value was obtained for each condition and participant. Finally, we used $t$ tests to compare the PSC values in the different task conditions. All $t$ tests were calculated one-tailed with an alpha level of $5 \%$ because of directed hypotheses about the expected effects.

\section{Acknowledgments}

We thank Anke Mempel, Mandy Naumann, Anke Pitzmaus, and Kathrin Wiesner for their assistance in data acquisition, and Hugh Garavan and an anonymous reviewer for their valuable comments on an earlier version of the manuscript.

Reprint requests should be sent to André Szameitat, MaxPlanck-Institute of Cognitive Neuroscience, PO Box 500 355, D-04303 Leipzig, Germany, or via e-mail: szameit@cns.mpg.de.

The data reported in this experiment have been deposited in The fMRI Data Center (http://www.fmridc.org). The accession number is $2-2002-1134 \mathrm{~F}$.

\section{Notes}

1. There is a recent controversy about whether this bottleneck is immutable or not (e.g., Levy \& Pashler, 2001; Schumacher et al., 2001). However, this controversy does not concern the assumption underlying the present study that executive functions are needed to coordinate task processing at the stage of the bottleneck.

2. We interpreted our design as $2 \times 2$ factorial design with the factors Auditory Task and Visual Task, both incorporating the levels Task Present and Task Absent. Both tasks absent constitutes the resting baseline condition, either auditory or visual task present the single-task conditions and both tasks present the dual-task condition. This enables to test for interaction between the factors by using the contrast ((DT-fixed-AUD) - (VIS-BASE)).

3. Although the activation in the right SFG in the condition VIS did not reach significance after Bonferroni correction for multiple comparisons ( $p=.000011$, uncorrected), we considered it for the purpose of this analysis.

\section{REFERENCES}

Adcock, R. A., Constable, R. T., Gore, J. C., \& Goldman-Rakic, P. S. (2000). Functional neuroanatomy of executive processes involved in dual-task performance. Proceedings of the National Academy of Sciences, U.S.A., 97, 3567-3572.

Baddeley, A. (1986). Working memory. Oxford, UK: Clarendon Press.

Baddeley, A. (1990). Human memory: Theory and practice. Hove, London: Erlbaum.

Baddeley, A. (1998). The central executive: A concept and some misconceptions. Journal of the International Neuropsychological Society, 4, 523-526.

Baddeley, A., \& Della Sala, S. (1996). Working memory and executive control. Philosophical Transactions of the Royal Society of London, Series B: Biological Sciences, 351, 1397-1404

Badgaiyan, R. D. (2000). Executive control, willed actions, and nonconscious processing. Human Brain Mapping, 9, 38-41.

Bosch, V. (2000). Statistical analysis of multi-subject fMRI data: The assessment of focal activations. Journal of Magnetic Resonance, 11, 61-64.

Braver, T. S., Cohen, J. D., Nystrom, L. E., Jonides, J., Smith, E. E., \& Noll, D. C. (1997). A parametric study of prefrontal cortex involvement in human working memory. Neuroimage, 5, 49-62.

Broadbent, D. E. (1982). Task combination and the selective intake of information. Acta Psychologica, 50, 253-290.

Bunge, S. A., Klingberg, T., Jacobsen, R. B., \& Gabrieli, J. D. E. (2000). A resource model of the neural basis of executive working memory. Proceedings of the National Academy of Sciences, U.S.A., 97, 3573-3578.

Callicott, J. H., Mattay, V. S., Bertolino, A., Finn, K., Coppola, R., Frank, J. A., Goldberg, T. E., \& Weinberger, D. R. (1999). Physiological characteristics of capacity constraints in working memory as revealed by functional MRI. Cerebral Cortex, 9, 20-26.

Cohen, J. D., Perlstein, W. M., Braver, T. S., Nystrom, L. E., Noll, D. C., Jonides, J., \& Smith, E. E. (1997). Temporal dynamics of brain activation during a working memory task. Nature, 386, 604-607.

Coull, J. T., \& Nobre, A. C. (1998). Where and when to pay attention: The neural systems for directing attention to spatial locations and to time intervals as revealed by both PET and fMRI. The Journal of Neuroscience, 18, 7426-7435.

Craik, K. W. J. (1947). Theory of the human operator in control systems: I. The operator as an engineering system. British Journal of Psychology, 38, 56-61.

DeJong, R. (1993). Multiple bottlenecks in overlapping task performance. Journal of Experimental Psychology: Human Perception and Performance, 19, 965-980. 
DeJong, R. (1995). The role of preparation in overlapping-task performance. Quarterly Journal of Experimental Psychology, 48A, 2-25.

D'Esposito, M., Detre, J. A., Alsop, D. C., Shin, R. K., Atlas, S., \& Grossman, M. (1995). The neural basis of the central executive system of working memory. Nature, 378, 279-281.

Donders, F. C. (1896/1969). On the speed of mental processes (W. G. Koster, Trans.). In W. G. Koster (Ed.), Attention and performance II (pp. 412-431). Amsterdam: North-Holland.

Dove, A., Pollmann, S., Schubert, T., Wiggins, C. J., \& von Cramon, D. Y. (2000). Prefrontal cortex activation in task switching: An event-related fMRI study. Cognitive Brain Research, 9, 103-109.

Friston, K. J., Holmes, A. P., Worsley, K. J., Poline, J.-P., Frith, C. D., \& Frackowiak, R. S. J. (1995). Statistical parametric maps in functional imaging: A general linear approach. Human Brain Mapping, 2, 189-210.

Friston, K. J., Price, C. J., Fletcher, P., Moore, C., Frackowiak, S. J., \& Dolan, R. J. (1996). The trouble with cognitive subtraction. Neuroimage, 4, 97-104.

Friston, K. J., Williams, S., Howard, R., Frackowiak, R. S. J., \& Turner, R. (1996). Movement-related effects in fMRI time-series. Magnetic Resonance in Medicine, 35, $346-355$.

Frith, C. D., \& Done, D. J. (1986). Routes to action in reaction time tasks. Psychological Research, 48, 169-177.

Goldberg, T. E., Berman, K. F., Fleming, K., Ostrem, J., VanHorn, J., Esposito, G., Mattay, V. S., Gold, J. M., \& Weinberger, D. R. (1998). Uncoupling cognitive workload and prefrontal cortical physiology: A PET rCBF study. Neuroimage, 7, 296-303.

Herath, P., Klingberg, T., Young, J., Amunts, K., \& Roland, P. (2001). Neural correlates of dual task interference can be dissociated from those of divided attention: An fMRI study. Cerebral Cortex, 11, 796-805.

Klingberg, T. (1998). Concurrent performance of two working memory tasks: Potential mechanisms of interference. Cerebral Cortex, 8, 593-601.

Knight, R. T., Grabowecky, M. F., \& Scabini, D. (1995). Role of human prefrontal cortex in attention control. In $\mathrm{H}$. H Jasper, S. Riggio, \& P. S. Goldman-Rakic (Eds.), Epilepsy and the functional anatomy of the frontal lobe (pp. 1357-1371). New York: Raven Press.

Koechlin, E., Basso, G., Pietrini, P., Panzer, S., \& Grafman, J. (1999). The role of the anterior prefrontal cortex in human cognition. Nature, 399, 148-151.

Konishi, S., Nakajima, K., Uchida, I., Kikyo, H., Kameyama, M., \& Miyashita, Y. (1999). Common inhibitory mechanism in human inferior prefrontal cortex revealed by event-related functional MRI. Brain, 122, 981-991.

Kray, J., \& Lindenberger, U. (2000). Adult age differences in task switching. Psychology and Aging, 15, 126-147.

Levy, J., \& Pashler, H. (2001). Is dual-task slowing instruction dependent? Journal of Experimental Psychology: Human Perception and Performance, 27, 862-869.

Loftus, G. R., \& Masson, M. E. J. (1994). Using confidence intervals in within-subject designs. Psychonomic Bulletin $E$ Review, 1, 476-490.

Lohmann, G., Mueller, K., Bosch, V., Mentzel, H., Hessler, S., Chen, L., Zysset, S., \& von Cramon, D. Y. (2001). Lipsiaa new software system for the evaluation of functional magnetic resonance images of the human brain. Computerized Medical Imaging and Graphics, 25, $449-457$.

MacDonald, A. W., III, Cohen, J. D., Stenger, V. A., \& Carter, C. S. (2000). Dissociating the role of the dorsolateral prefrontal and anterior cingulate cortex in cognitive control. Science, 288, 1835-1838.
Meyer, D. E., \& Kieras, D. E. (1997). A computational theory of executive cognitive processes and multiple-task performance: Part 1. Basic mechanisms. Psychological Review, 104, 3-65.

Miller, E. K. (2000). The prefrontal cortex and cognitive control. Nature Reviews: Neuroscience, 1, 59-65.

Oldfield, R. C. (1971). The assessment and analysis of handedness: The Edinburgh Inventory. Neuropsychologia 9, 97-113.

Pashler, H. (1994). Dual-task interference in simple tasks: Data and theory. Psychological Bulletin, 116, 220-244.

Petrides, M., \& Pandya, D. N. (1999). Dorsolateral prefrontal cortex: Comparative cytoarchitectonic analysis in the human and the macaque brain and corticocortical connection patterns. European Journal of Neuroscience, 11, 1011-1036.

Romanski, L. M., Tian, B., Fritz, J., Mishkin, M., Goldman-Rakic, P. S., \& Rauschecker, J. P. (1999). Dual streams of auditory afferents target multiple domains in the primate prefrontal cortex. Nature Neuroscience, 2, 1131-1135.

Rowe, J. B., Toni, I., Josephs, O., Frackowiak, R. S. J., \& Passingham, R. E. (2000). The prefrontal cortex: Response selection or maintenance within working memory? Science, 288, 1656-1660.

Rypma, B., Prabhakaran, V., Desmond, J. E., Glover, G. H., \& Gabrieli, D. E. (1999). Load-dependent roles of frontal brain regions in the maintenance of working memory. Neuroimage, 9, 216-226.

Sartori, G., \& Umiltà, C. (2000). How to avoid the fallacies of cognitive subtraction in brain imaging. Brain and Language, $74,191-212$

Schubert, T. (1999). Processing differences between simple and choice reaction affect bottleneck localization in overlapping tasks. Journal of Experimental Psychology: Human Perception and Performance, 25, $408-425$.

Schubert, T., von Cramon, D. Y., Niendorf, T., Pollmann, S., \& Bublak, P. (1998). Cortical areas and the control of self-determined finger movements: An fMRI study. NeuroReport, 9, 3171-3176.

Schubotz, R. I., Friederici, A. D., \& von Cramon, D. Y. (2000). Time perception and motor timing: A common cortical and subcortical basis revealed by fMRI. Neuroimage, 11, $1-12$.

Schubotz, R. I., \& von Cramon, D. Y. (2001). Interval and ordinal properties of sequences are associated with distinct premotor areas. Cerebral Cortex, 11, 210-222.

Schumacher, E. H., Seymour, T. L., Glass, J. M., Fencsik, D. E., Lauber, E. J., Kieras, D. E., \& Meyer, D. E. (2001). Virtually perfect time sharing in dual-task performance: Uncorking the central cognitive bottleneck. Psychological Science, 12, $101-108$.

Shima, K., \& Tanji, J. (1998). Both supplementary and presupplementary motor areas are crucial for the temporal organization of multiple movements. Journal of Neurophysiology, 80, 3247-3260.

Shima, K., \& Tanji, J. (2000). Neuronal activity in the supplementary and presupplementary motor areas for temporal organization of multiple movements. Journal of Neurophysiology, 84, 2148-2160.

Sidtis, J. J., Strother, C., Anderson, J. R., \& Rottenberg, D. A. (1999). Are brain functions really additive? Neuroimage, 9 , 490-496.

Smith, E. E., Geva, A., Jonides, J., Miller, A., Reuter-Lorenz, P., \& Koeppe, R. A. (2001). The neural basis of task-switching in working memory: Effects of performance and aging. Proceedings of the National Academy of Sciences, U.S.A., 98, $2095-2100$ 
Sohn, M.-H., Ursu, S., Anderson, J. R., Stenger, A., \& Carter, C. S. (2000). The role of prefrontal cortex and posterior parietal cortex in task switching. Proceedings of the National Academy of Sciences, U.S.A., 97, 13448-13453. Sternberg, S. (1969). The discovery of processing stages: Extensions of Donders' method. Acta Psychologica, 30, $276-315$.

Talairach, J., \& Tournoux, P. (1988). Co-planar stereotaxic atlas of the buman brain. New York: Thieme.

Umiltà, C., Nicoletti, R., Simion, F., Tagliabue, M. E., \& Bagnara,
S. (1992). The cost of a strategy. European Journal of Cognitive Psychology, 4, 21-40.

Wojciulik, E., \& Kanwisher, N. (1999). The generality of parietal involvement in visual attention. Neuron, 23, $747-764$.

Worsley, K. J., \& Friston, K. J. (1995). Analysis of fMRI time-series revisited-again. Neuroimage, 2, 173-181.

Zysset, S., Müller, K., Lohmann, G., \& von Cramon, D. Y. (2001). Color-word matching Stroop task: Separating interference and response conflict. Neuroimage, 13, 29-36. 